ISSN: 2602-8506

Vol. 3, N², p. 26-46, abril - junio, 2019

\title{
Evaluación de la calidad del servicio de transporte urbano en bus de la ciudad de Ambato
}

\author{
Evaluation of the quality of the urban transport service by bus of the city of \\ Ambato \\ José Luis Llamuca Llamuca. ${ }^{1}$ \& Gustavo Javier Aguilar Miranda. ${ }^{2}$
}

DOI: https://doi.org/10.33262/visionariodigital.v3i2.392

\section{Resumen.}

Esta investigación tuvo como objetivo evaluar la calidad del servicio de transporte urbano en bus de la ciudad de Ambato, medida desde la perspectiva del cliente. Este estudio se fundamentó en un enfoque cualitativo, con un proceso deductivo y analizó la realidad objetiva, el alcance fue descriptivo y correlacional, se trabajó con una muestra de 384 usuarios del servicio de transporte, se combinaron los métodos analítico y sintético. Los resultados alcanzados, han permitido visualizar el nivel de satisfacción de los usuarios del servicio de transporte urbano de Ambato, considerando variables como: Servicio ofertado, Accesibilidad, Información, Tiempo, Atención al cliente, Confort, Seguridad e Impacto ambiental, concluyendo que en las ocho variables estudiadas el nivel de satisfacción esta, por debajo de 4 sobre 10; lo que implica que los usuarios están insatisfechos con el servicio de transporte urbano en bus en la ciudad de Ambato, por lo tanto le corresponde al GAD de Ambato, apoderarse de la situación, y se emprenda en procesos de solución inmediatos, reprogramando rutas, y brindando mayor cobertura, así como también con el estudio de la flota vehicular, adecuación de vías y señales de tránsito y por otro lado exigiendo a las operadoras de transporte urbano en bus la corrección de sus procesos para enmendar las falencias detectadas en el servicio que prestan.

Palabras claves: transporte urbano, bus, calidad, servicio, evaluación.

\footnotetext{
${ }^{1}$ Escuela Superior Politécnica de Chimborazo, Facultad de Administración de Empresas, Riobamba, Ecuador, jose.1lamuca@espoch.edu.ec

2 Escuela Superior Politécnica de Chimborazo, Facultad de Administración de Empresas, Riobamba Ecuador,gustavo.aguilar@espoch.edu.ec
} 


\section{Abstract.}

The objective of this research was to evaluate the quality of the urban bus service in the city of Ambato, measured from the customer's perspective. This study was based on a qualitative approach, with a deductive process and analyzed the objective reality, the scope was descriptive and correlational, we worked with a sample of 384 users of the transport service, the analytical and synthetic methods were combined. The results achieved have allowed to visualize the level of satisfaction of the users of the urban transport service of Ambato, considering variables such as: Service offered, Accessibility, Information, Time, Customer service, Comfort, Safety and Environmental impact, concluding that in the eight variables studied, the satisfaction level is below 4 out of 10; which implies that users are dissatisfied with the urban bus service in the city of Ambato, therefore it is up to Ambato's GAD to take charge of the situation, and undertake immediate solution processes, reprogramming routes, and providing greater coverage, as well as the study of the vehicle fleet, adaptation of roads and traffic signals and on the other hand requiring bus operators to correct their processes to correct the shortcomings detected in the service they provide.

Keywords: Urban transport, bus, quality, service, evaluation.

\section{Introducción.}

El transporte tiene relación muy estrecha con el desarrollo económico del país, así los servicios de transporte son importantes para el desarrollo de la sociedad, ya que el mismo hace posible el acceso a recursos, bienes, insumos etc. que de otra manera no serían asequibles por razones de distancia.

Por todo esto se puede considerar al transporte como el motor que dinamiza la economía de cualquier ciudad o país en general, en nuestro país el sector del transporte y almacenamiento representa aproximadamente un 7\% del PIB (Hubenthal, 2010). Pero durante las últimas décadas ha tenido grandes problemas en el sistema de transporte público urbano para satisfacer las necesidades de la población que es de 14.306.876 habitantes, según los datos del último censo poblacional del Instituto Nacional de Estadísticas y Censo. (INEC, 2010)

Por otra parte, el desmedido crecimiento del automóvil en el país ha provocado que la mayor parte de la población se transporte por este medio. Esto puede ser denotado en las cifras emitidas por el Ministerio de Transporte y Obras Públicas (MTOP) que indica que en 1990 había un total de 320.000 vehículos matriculados, en el año 1998 eran unos 590.000 y en el 2008 eran aprox. 990.000. Según el MTOP (Publicas, 2009), el crecimiento ha sido más fuerte por lado de los vehículos destinados para el transporte de personas en 
comparación a los vehículos para el transporte de carga, de ahí puede determinarse que ha existido un incremento significativo en relación a la movilidad individual.

Ya en el contexto local, la ciudad de Ambato cuenta con una población según el INEC (Censos, 2010) de 382.941 habitantes, ubicándose en la quinta cantón más poblado del Ecuador. Esta población es atendida por un sistema de transporte público urbano que está conformado por cinco empresas privadas con una flota de 433 buses convencionales, dando cobertura con un total de 22 rutas que permiten la movilidad de los ambateños en las nueve parroquias urbanas de la ciudad y diez parroquias rurales.

Actualmente el sistema de transporte público urbano en la ciudad de Ambato, al igual que en la gran mayoría de las ciudades medias y pequeñas del país presenta graves problemas en la prestación del servicio, tanto en la parte operacional como en la administración del servicio no hay una estructura adecuada que permita brindar un servicio público de calidad, ello ha ocasionado que este servicio sea lento, inseguro, contaminante y por el otro lado quienes prestan el mismo tengan una baja rentabilidad financiera.

Además, en la actualidad, otro factor que complica al sistema de transporte público urbano de Ambato es la competencia aguda generada por el mayor uso del automóvil privado, ya que partir del año 2000 los bajos porcentajes de inflación y el esquema de dolarización permitieron recuperar el poder de compra de la población. A partir de 2007 el incremento del salario real aumenta en velocidad (BCE, 2013), acompañado este escenario a la facilidad de acceso al crédito ocasiono una creciente motorización lo que provocó menores ingresos para las empresas privadas prestadoras del servicio.

Por último, la transferencia de las competencias de transporte y tránsito del país hacia los Municipios sin un análisis correcto de la capacidad y experiencia de los nuevos administradores del transporte ha ocasionado que no se defina políticas claras respecto al manejo de los recursos para fortalecer y definir estrategias que vayan a mejorar al sistema de transporte público urbano.

En este sentido, la eficacia y la eficiencia del transporte urbano representan un reto trascendental. Es necesario proporcionar un transporte público eficaz, rápido, seguro y económico, a la vez que paliar o cuando menos minimizar los problemas de congestión y coordinación de tráfico (Sánchez, Carrasco, y Sánchez, 2014).

Frente a esta problemática es relevante la investigación a realizarse para medir la calidad del servicio que ofertan las operadoras del sistema de transporte urbano, para mejorar las condiciones en la entrega del servicio de transporte público urbano hacia la ciudad de Ambato. 


\section{Desarrollo.}

En la actualidad, el sistema de transporte público urbano ocupa un papel esencial en la vertebración económica y social de las ciudades y núcleos poblacionales de todo el mundo y también en nuestro país.

El crecimiento poblacional genera necesidades básicas a ser cubiertas por quienes dirigen los pueblos, los sistemas de urbanización que se originan en función de los requerimientos de los habitantes de una ciudad trae como consecuencia la necesidad de movilidad, al encontrarse las instituciones educativas los comercios la banca así como los organismos estatales generalmente en la zona central de las ciudades obliga a que la población en busca de acceder a los servicios que brindan estas instituciones provoca un movimiento masivo diario de dichas personas.

La limitada calidad en la prestación del servicio de movilidad, que a lo largo del tiempo han proporcionado los actores de este sistema, unido a la creciente demanda, ha provocado en las autoridades locales la búsqueda de alternativas hacia una mejor operatividad del servicio.

En este sentido, un trabajo significativo es el de Silva y Torres en su tesis "Calidad del servicio de transporte urbano en la ciudad de Cuenca", el mismo que tiene como objetivo determinar los factores preponderantes de la calidad del transporte urbano de pasajeros, $\mathrm{y}$ presenta una propuesta para el mejoramiento de la calidad del servicio del transporte urbano de la ciudad de Cuenca (Silva \& Torres, 2017).

Gabriel Morán, en su tesis: "Análisis de la calidad del servicio de transporte urbano de buses en la ciudad de Esmeraldas desde la óptica del usuario”, ejecutado en 2016, concluye que la calidad del servicio de transporte urbano en la ciudad de esmeraldas presenta múltiples deficiencias debido a la falta de evaluaciones al servicio en casi sesenta años de utilización, siendo el trato recibido por parte de los choferes y cobradores la mayor queja que presentan los usuarios. (Morán, 2016)

\section{Gestión de la Movilidad.}

Desde el punto de vista organizativo, se puede afirmar que cada vez es mayor la relación e interdependencia entre la gran ciudad y los municipios dentro de su área de influencia, por lo que se debe prestar especial atención al transporte urbano, interurbano o metropolitano. En palabras de Giménez y Capdevila (2004, pág.2), el sistema de transporte desarrolla sus estructuras técnicas sobre un territorio fragmentado institucionalmente, de manera que los ámbitos territoriales de las redes de transporte y de las instituciones político-administrativas que ejercen el poder no siempre coinciden. Esto es más evidente a escala urbana y regional 
puesto que los patrones de movilidad han cambiado de manera muy significativas en las últimas décadas, mientras que los reajustes institucionales han sido mínimos.

Asimismo, el transporte tiene un papel fundamental en el crecimiento económico de las ciudades, no sólo por la importancia del sector en sí mismo (personas empleadas, productividad, etc.) sino por ser una actividad complementaria al resto de los sectores (industria, comercio, turismo) y por su impacto medioambiental y social de las ciudades (Arroyo, 2008, pág. 51).

El momento de la planificación urbanística es el período idóneo para el cálculo de todas las redes, de abastecimientos, redes de eliminación de residuos y, cómo no, las redes de movilidad. En concreto, habrá que identificar la movilidad que se generará estudiando conjuntamente los modos de transporte existentes: peatones, bicicletas, los distintos tipos de transporte público, vehículos privados y mercancías. Todo lo que no sea un diseño a priori de las redes de movilidad, llevará consigo un aumento de los costes en solucionar los problemas y efectos que no se hayan calculado con anterioridad (Dombriz, 2009, págs. 49).

Así pues, el diseño de las ciudades debe ser resultado de la planificación urbanística y estar directamente relacionado con la movilidad de viajeros y mercancías que se desarrollan en ellas.

No serán iguales los desplazamientos de personas y mercancías en las ciudades donde los centros de trabajo, ocio y estudios estén concentrados con un alto nivel de densidad, que los núcleos urbanos donde los usos están más distanciados.

En el primer tipo de ciudades las necesidades de movilidad vienen satisfechas por un transporte público eficiente y por continuos desplazamientos a pie o en bicicleta. Por el contrario, las ciudades dispersas han dado lugar a un modelo de movilidad caracterizado por el uso excesivo de los vehículos privados, a veces por necesidad ya que no hay transporte sustitutivo, o bien por comodidad, ya que la primera variable es el tiempo utilizado en los desplazamientos.

El modelo elegido en la mayoría de las ciudades ecuatorianas se basa en la idea de segregación: centros donde se hacen oficinas, polígonos industriales, grupos de viviendas, centros comerciales y de ocio, etc., donde el medio de transporte por excelencia es el vehículo particular. No existen ningún tipo de limitaciones ni para el uso ni para el consumo de los combustibles derivados del petróleo. El abuso en el uso del coche propio lleva consigo una serie de impactos o externalidades en la colectividad. Estos impactos son, en su mayoría, costes que la sociedad debe soportar (congestión, contaminación y 
ocupación del suelo, entre otras) y que, por tanto, se hace necesaria su consideración y valoración.

\section{Calidad del Servicio.}

Para definir la calidad de las empresas y su gestión en los servicios que prestan, es importante analizarla desde la perspectiva del usuario, es decir cuál es la opinión que él tiene respecto de los servicios en este caso que la empresa oferta y así conocer si sus necesidades están satisfechas.

Al respecto, (Domínguez H. , 2006) considera que la calidad tiene que ver con la fiabilidad que se tiene hacia una empresa, cuando ésta presta un servicio. En este sentido, la confianza que el cliente tiene respecto de la empresa determina si existe o no calidad en el servicio ofertado.

Gutiérrez, explica que para entender el concepto de calidad de servicio se deben conocer primero los conceptos de calidad, servicio y cliente por separado. Se define calidad como la totalidad de funciones, características o comportamientos de un bien o servicio; volverá a elegirlo tantas veces como lo precise en el transcurso de su vida, por lo que el tener una calidad en el presente influye en la visión que se forja de la organización en el futuro, sobre todo en el área de servicios, donde lo que se ofrece es un bien intangible, en consecuencia lo que influye es la idea que nuestros clientes y potenciales clientes tengan del servicio ofrecido. (Gutiérrez, 2010).

UUna visión actual del concepto de calidad indica que calidad es entregar al cliente no lo que quiere, sino lo que nunca se había imaginado que quería y que una vez que lo obtenga, se dé cuenta que era lo que siempre había querido. (Gutiérrez, 2008)

Siguiendo la misma línea, la Norma Europea UNE-EN 13816 sobre calidad en el transporte público de pasajeros (Fundación CETMO, 2006) menciona que la calidad del servicio objetivo es el nivel de calidad que el operador y el titular del servicio tienen por objetivo ofrecer a los clientes, se define en función del nivel de calidad esperado por los clientes.

\section{El transporte de calidad}

El transporte público servicio fundamental en nuestra sociedad es una de las actividades más directamente relacionadas con la calidad de vida de los ciudadanos la norma europea EN 13816 (Ver Tabla 1) de transporte público de pasajeros proporciona directrices a los operadores para que sea de calidad en la prestación de sus servicios con el fin de satisfacer las expectativas de sus clientes los compromisos de calidad deben asumirse en ocho 
ámbitos: tiempo, confort, información, accesibilidad, seguridad, servicio ofertado, atención al cliente, e impacto ambiental. (Comité Europeo de Normalización, 2006)

Tabla 1. Criterios de calidad según la norma UNE-EN 13816.

\begin{tabular}{|c|c|}
\hline \multicolumn{2}{|c|}{ CRITERIOS DE CALIDAD DE LA NORMA UNE-EN 13816} \\
\hline Nivel 1 & Nivel 2 \\
\hline \multirow{5}{*}{ 1. Servicio Ofertado } & Modos de transporte \\
\hline & Red \\
\hline & Explotación \\
\hline & Adecuación a las necesidades \\
\hline & Fiabilidad del servicio \\
\hline \multirow{3}{*}{ 2. Accesibilidad } & Accesibilidad externa \\
\hline & Accesibilidad interna \\
\hline & Expedición/adquisición de títulos de transporte \\
\hline \multirow{3}{*}{ 3. Información } & Información general \\
\hline & Información relativa al viaje en condiciones normales \\
\hline & $\begin{array}{l}\text { Información relativa al viaje en condiciones } \\
\text { anormales }\end{array}$ \\
\hline \multirow{2}{*}{ 4. Tiempo } & Duración del viaje \\
\hline & Cumplimiento de horarios \\
\hline \multirow{5}{*}{ 5. Atención al cliente } & Compromiso \\
\hline & Relación con los clientes \\
\hline & Personal \\
\hline & Asistencia \\
\hline & Adquisición de títulos de transporte \\
\hline \multirow{6}{*}{ 6. Confort } & Funcionalidad del equipamiento \\
\hline & Asientos y espacios para los pasajeros \\
\hline & Confort del viaje \\
\hline & Condiciones ambientales \\
\hline & Instalaciones complementarias \\
\hline & Ergonomía \\
\hline \multirow{3}{*}{ 7. Seguridad } & Protección contra agresiones \\
\hline & Prevención de accidentes \\
\hline & Gestión de situaciones de emergencia \\
\hline \multirow{3}{*}{ 8. Impacto ambiental } & Contaminación \\
\hline & Recursos naturales \\
\hline & Infraestructuras \\
\hline
\end{tabular}

Fuente: (Comité Europeo de Normalización, 2006) 


\section{Metodologia.}

La metodología de trabajo aplicada en la presente investigación se basa principalmente en la Norma Europea UNE-EN 13816 sobre calidad en el transporte público de pasajeros (Fundación CETMO, 2006), con la aplicación de medidas estadísticas que permitan identificar, determinar, promediar, agrupar y condensar la información en parámetros o estimadores a todas las variables que contiene la norma en esta modalidad de servicio.

Para ello se estableció las siguientes etapas:

- Recopilación de datos en campo e información primaria

- Tabulación y procesamiento de la información

- Análisis de los resultados

Cada una de estas etapas contempló varias actividades específicas que permiten obtener ya sea una tendencia o la información respectiva de una o más variables incidentes en la investigación realizada.

El tamaño de muestra de acuerdo con las recomendaciones nacionales e internacionales (MTOP, 2010) se determinó a partir de la siguiente ecuación:

$$
\boldsymbol{n}=\frac{Z^{2} * N * p * q}{E^{2}(N-1)+Z^{2} * p * q}
$$

La muestra calculada con los datos correspondientes a la población del cantón Ambato de 382.941 habitantes, de acuerdo con la fórmula aplicada fue de 384 encuestas, las cuales se aplicaron a los usuarios del servicio de transporte urbano en bus de las nueve parroquias urbanas y diez parroquias rurales de la ciudad de Ambato, los mismos que se movilizan en las cinco operadoras de transporte urbano de la ciudad: Cooperativa Tungurahua, Unión Ambateña, Libertadores, Jerpazol, Vía Flores de la ciudad.

La tabulación y procesamiento de la información se lo realizo en tablas y gráficos con la ayuda del Programa Microsoft Excel.

\section{Resultados.}

\section{Evaluación de la calidad del servicio}

El objetivo del presente estudio es evaluar la calidad del servicio de transporte urbano en bus de la ciudad de Ambato, desde la percepción ciudadana, para lo cual se considera los Criterios de calidad según la norma europea UNE-EN 13816 cuyo fin es satisfacer las expectativas de los clientes, por lo tanto, la calidad debe analizarse en ocho ámbitos: 
tiempo, confort, información, accesibilidad, seguridad, servicio ofertado, atención al cliente, e impacto ambiental.

Los resultados de la investigación arrojaron los siguientes datos:

\section{Servicio ofertado.}

Pregunta 1. Utiliza usted el transporte urbano en bus.

Gráfico 1. Utiliza usted el transporte urbano en bus.

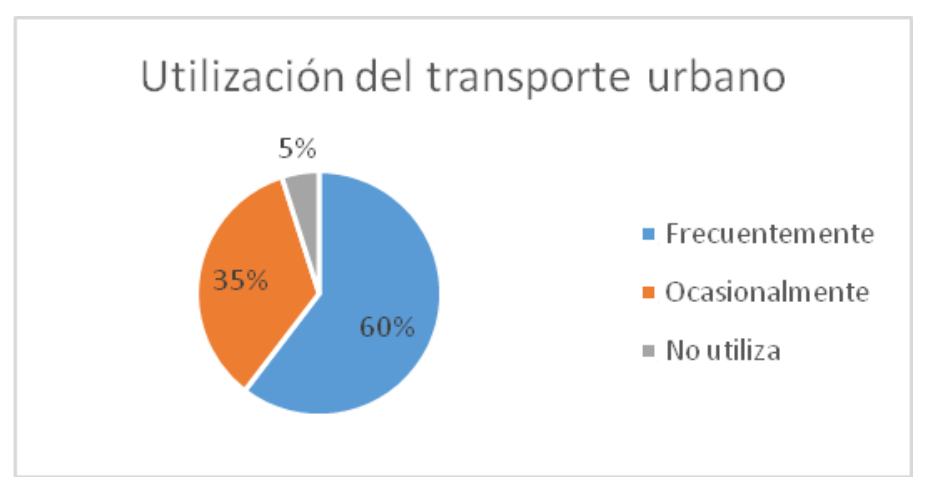

Fuente: Elaboración propia.

Análisis: Del total de encuestados, el servicio de transporte en bus lo utilizan un $60 \%$ frecuentemente, mientras que el 35 por ciento lo hace ocasionalmente, y el $5 \%$ no lo utiliza, lo que demuestra que la mayoría de población hace uso de este sistema para movilizarse independientemente de que el servicio le represente mayor tiempo de traslado coma así como los consecuentes riesgos, pues a decir de los encuestados el servicio no prestan la seguridad necesaria, por lo que parte de la población ha optado por la compra de su vehículo particular agravando la condición de movilidad.

Pregunta 2. Considera que la cobertura del transporte urbano en bus es:

Gráfico 2. Cobertura del transporte urbano bus.

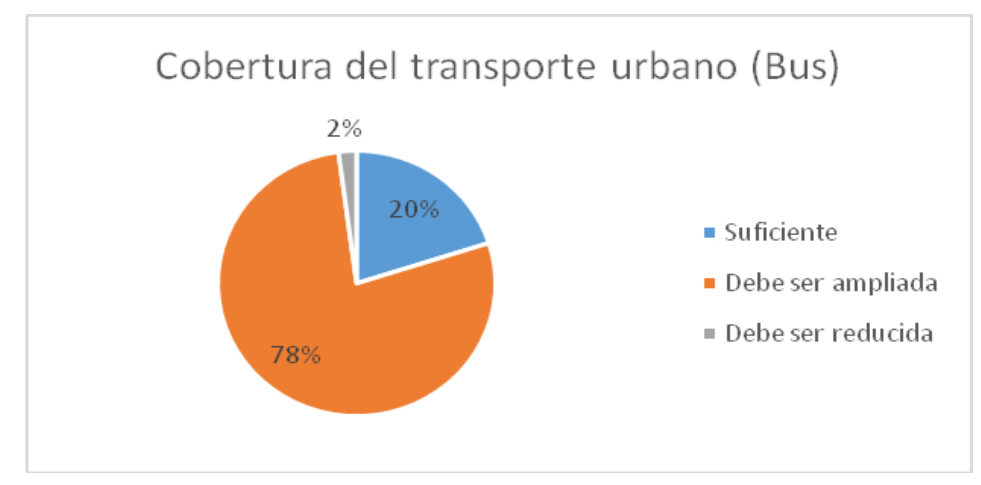

Fuente: Elaboración propia. 
Análisis: La percepción ciudadana con respecto a la cobertura del servicio, en un $78 \%$ consideran qué debe ser ampliada, el $20 \%$ manifiestan que es suficiente y el $8 \%$ qué debe ser reducida. La ciudad permanentemente crece, consecuentemente las urbanizaciones han generado la necesidad de movilización, por lo que requiere de una mayor cobertura hacia estas zonas que por lo general son lejanas, por lo tanto, es necesario que se reprograme las rutas ya que muchos ciudadanos deben tomar 2 o más buses para llegar a sus destinos.

Pregunta 3. El número de unidades que brindan el servicio de transporte es:

Gráfico 3. Número de unidades.

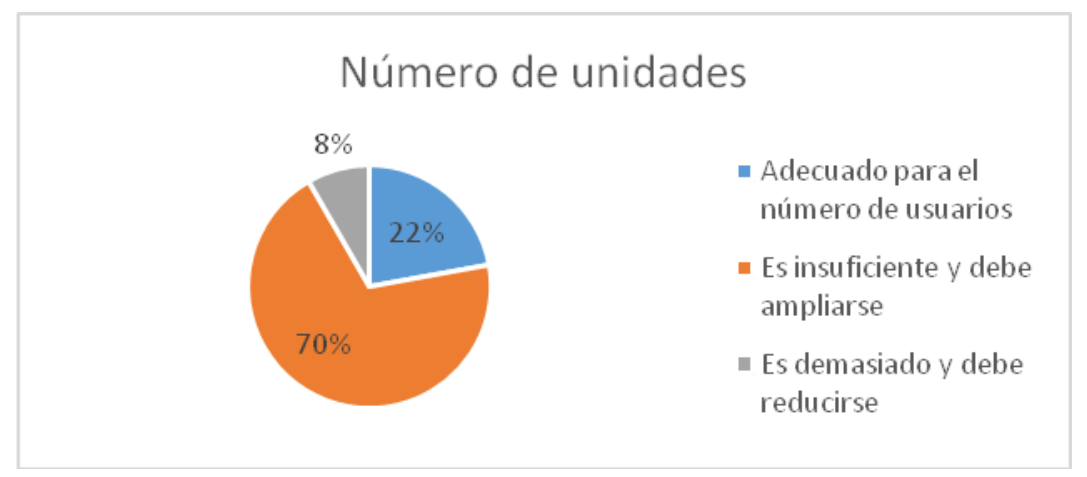

Fuente: Elaboración propia.

Análisis: La percepción ciudadana con respecto al número de unidades que brindan el servicio de transporte urbano, en un $70 \%$ consideran qué es insuficiente y debe ser ampliado, el $22 \%$ manifiestan que es el adecuado para el número de usuarios, y el $8 \%$ cree que es demasiado y debe reducirse.

Pregunta 4. Las Rutas que usted toma generalmente están:

Gráfico 4. Diseño de ruta.

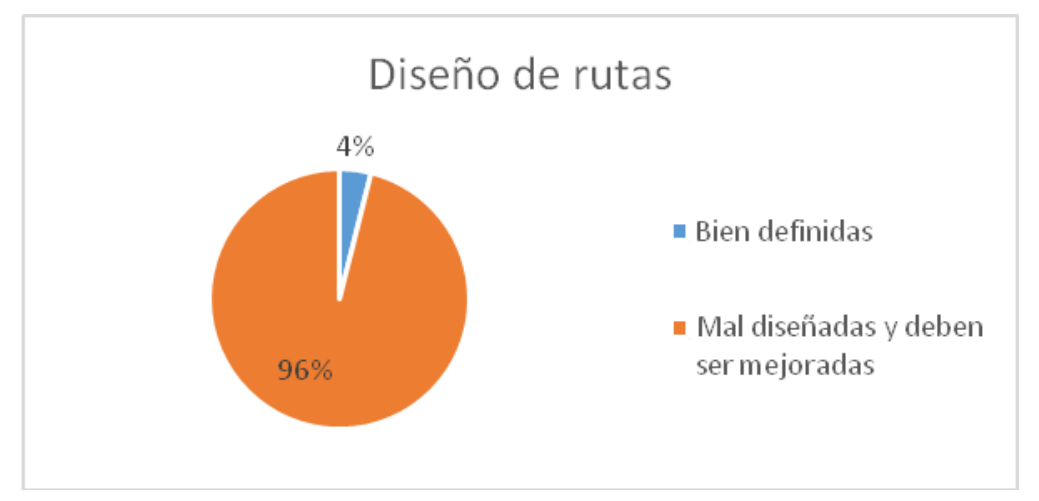

Fuente: Elaboración propia. 
Análisis: Del total de encuestados, el $96 \%$ manifiestan que las rutas están mal diseñadas y deben ser mejoradas y el $4 \%$ dice que están bien definidas.

Pregunta 5. La tarifa que paga por el uso del servicio de transporte urbano en Bus es:

Gráfico 5. Tarifa de pago por el uso del servicio de transporte.

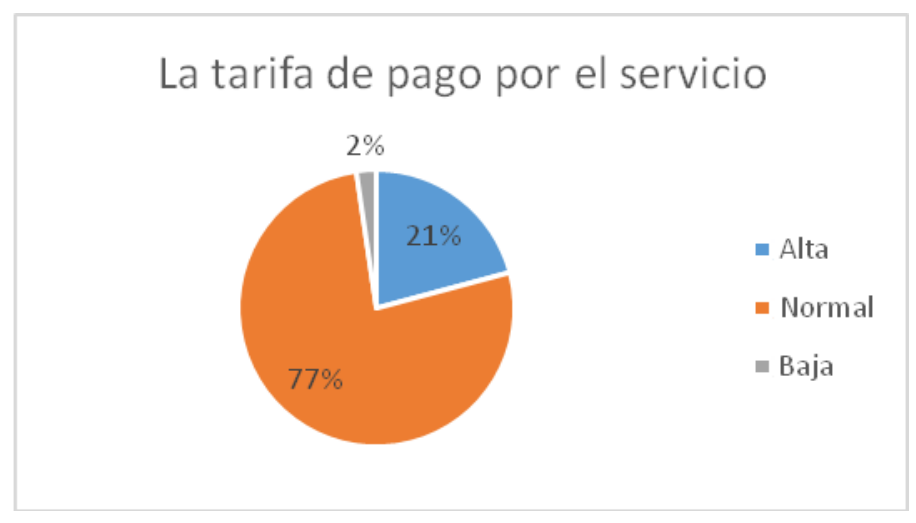

Fuente: Elaboración propia.

Análisis: El 77\% de encuestados, manifiesta que la tarifa es normal y está bien definida, el $21 \%$ manifiesta que la tarifa es alta y debe modificarse, y el $3 \%$ piensa que es baja y que se debería subir.

\section{Accesibilidad}

Pregunta 6. La accesibilidad que proporcionan los buses, para niños, mujeres embarazadas, adultos mayores y discapacitados es:

Gráfico 6. Inclusión a grupos vulnerables.

\section{Accesibilidad para grupos vulnerables}

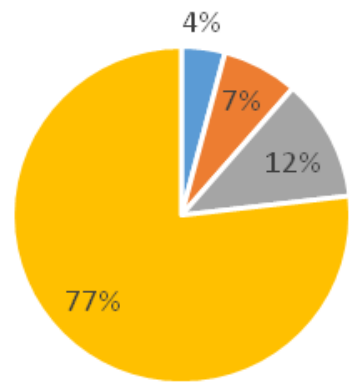

$$
\begin{aligned}
& \text { - Muy bueno } \\
& \text { - Bueno } \\
& \text { - Regular } \\
& \text { - Malo }
\end{aligned}
$$

Fuente: Elaboración propia. 
Análisis: Del total de encuestados, el $77 \%$ manifiestan que la accesibilidad para grupos vulnerables en los buses es mala, o que no existe, el $12 \%$ considera que es regular, el $7 \%$ considera que si es buena la accesibilidad que brindan las unidades de transporte, y el $4 \%$ cree que es muy bueno.

\section{Información.}

Pregunta 7. La información relativa al viaje, que le proporcionan en las paradas y en los buses es:

Gráfico 7. Información sobre el viaje en las paradas y buses.

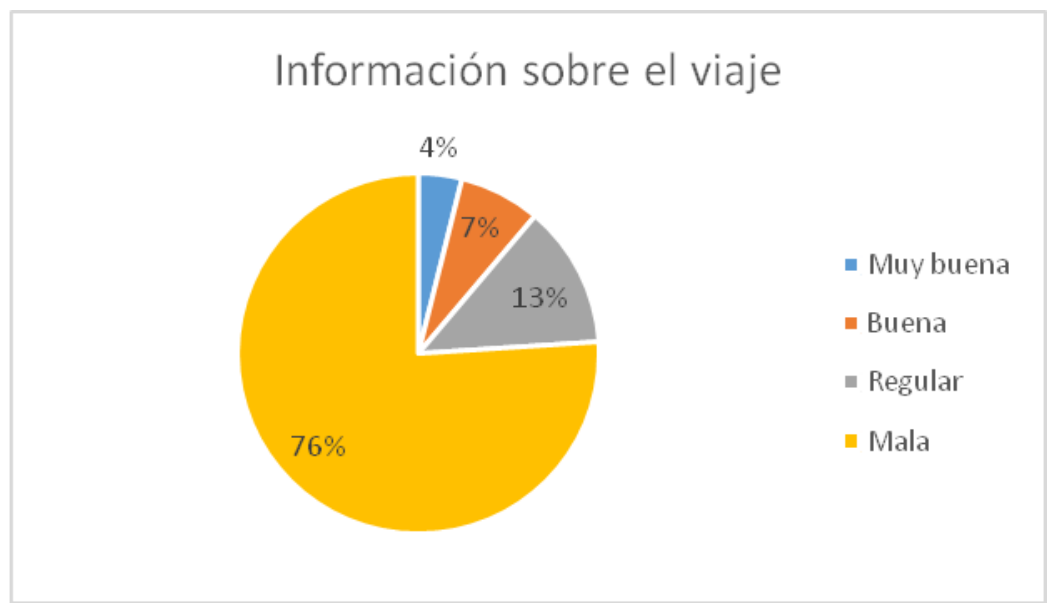

Fuente: Elaboración propia.

Análisis: Del total de encuestados, el $76 \%$ responde que la información relativa al viaje en paradas y buses es mala, el 13\% manifiesta que es regular, el $7 \%$ que es buena y el $4 \%$ que es muy buena.

\section{Tiempo.}

Pregunta 8. El tiempo que tarda en su recorrido (Ruta) desde su origen a su destino es:

Gráfico 8. Tiempo en ruta.

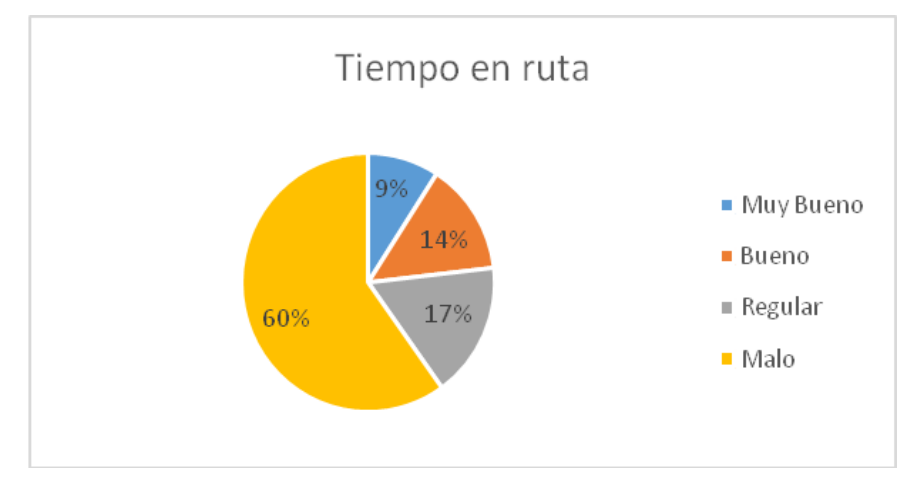


Fuente: Elaboración propia.

Análisis: El $60 \%$ de encuestados, manifiestan el tiempo en ruta es malo o que se demora mucho, el $17 \%$ manifiestan que es regular, el $14 \%$ que es un buen tiempo y el $9 \%$ que el tiempo en ruta es muy bueno o relativamente rápido.

\section{Atención al cliente.}

Pregunta 9. El trato recibido por parte de choferes y cobradores es:

Gráfico 9. Calificación del trato recibido por parte de choferes y cobradores.

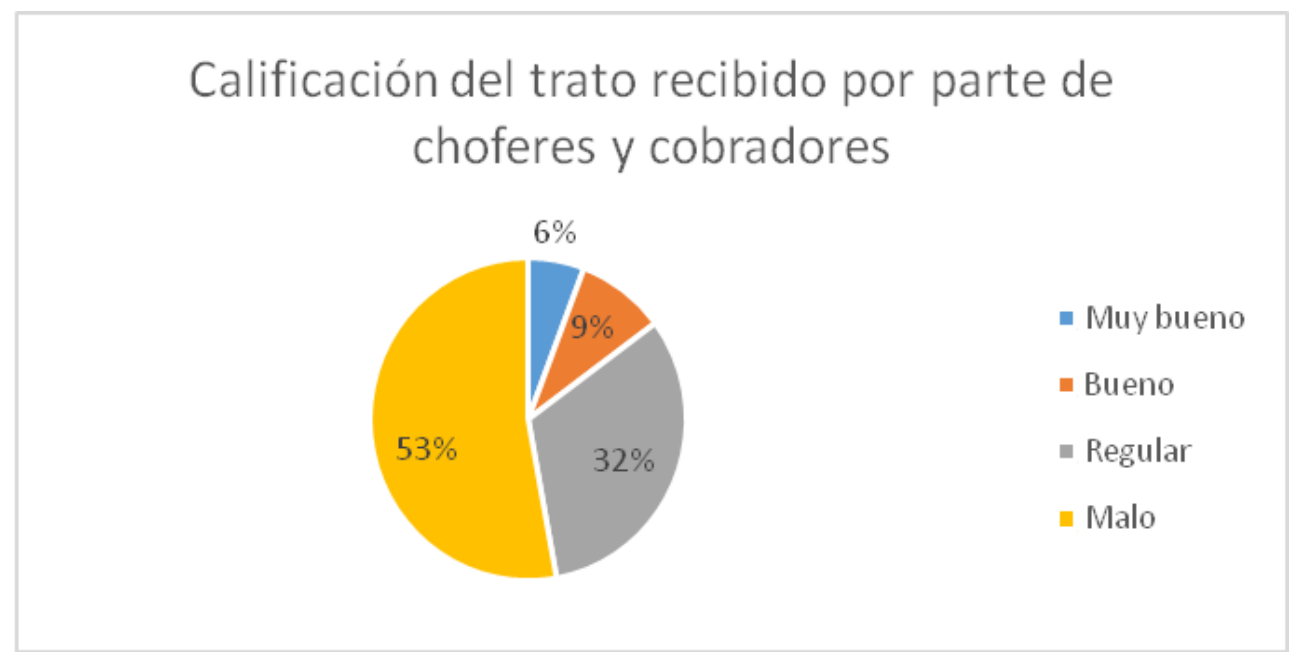

Fuente: Elaboración propia

Análisis: Del total de encuestados, el 53\% manifiestan que el trato recibido por parte de los choferes y cobradores es malo, el $32 \%$ cree que es regular, el $9 \%$ que es bueno y el $6 \%$ que es muy bueno.

Pregunta 10. Beneficios que quisieran obtener los usuarios del servicio de transporte en Bus:

Gráfico 10. Expectativas del usuario. 


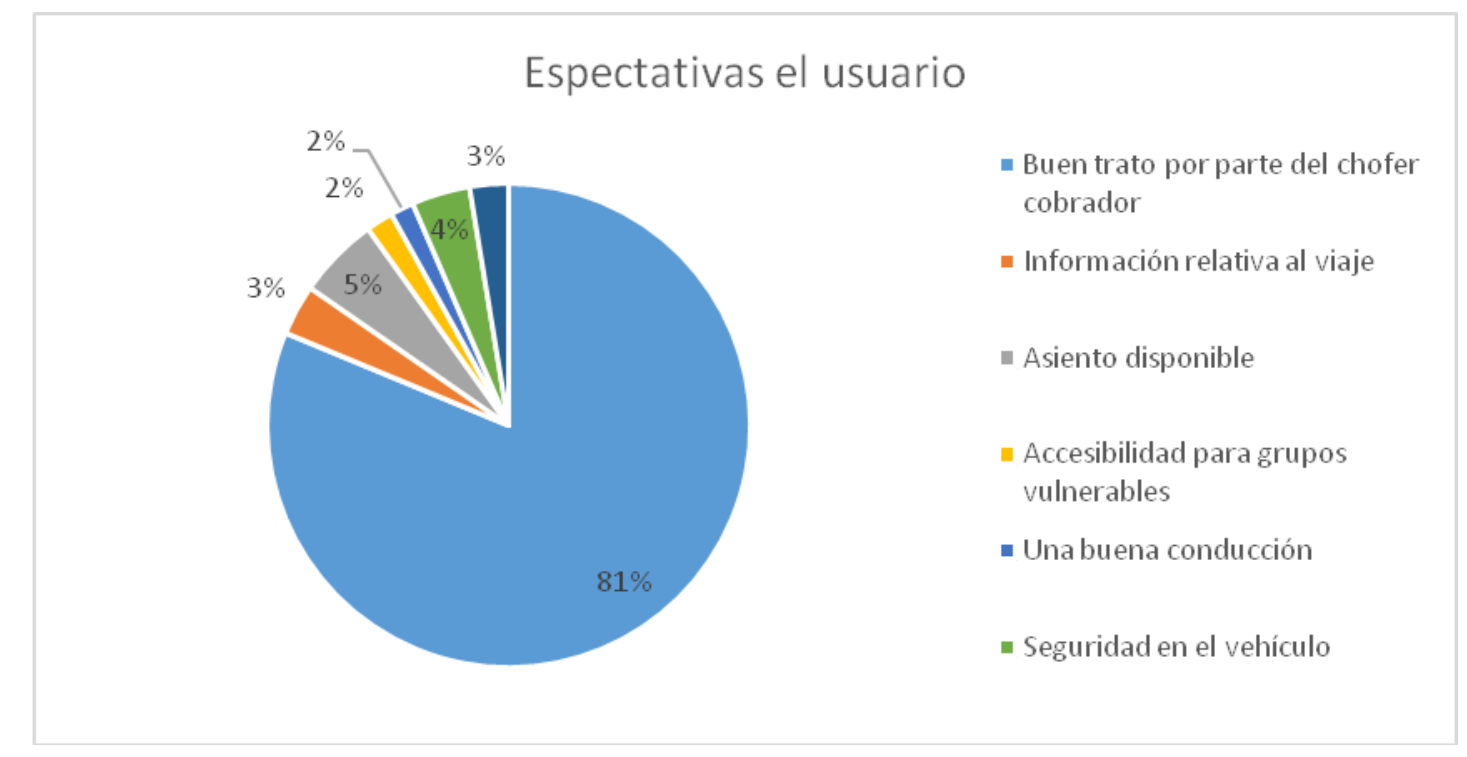

Fuente: Elaboración propia

Análisis: Del total de encuestados, el $81 \%$ manifiestan que el buen trato es el criterio más valorado por los usuarios del sistema de transporte, luego con un 5\% valoran el tener un asiento disponible, el $4 \%$ requieren seguridad, el 3\% información relativa al viaje, el $2 \%$ requieren Accesibilidad para grupos vulnerables y el último $2 \%$ solicitan una buena conducción de la unidad de transporte.

\section{Confort}

Pregunta 11. El confort que prestan las unidades de servicio de transporte a los pasajeros es:

Gráfico 11. El confort que prestan las unidades de servicio.

\section{El confort de las unidades de servicio}

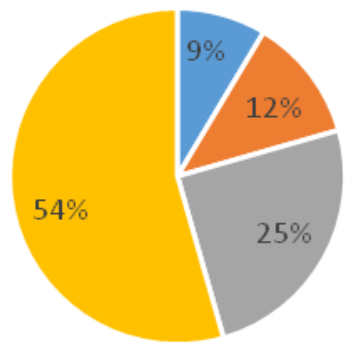

$$
\begin{aligned}
& \text { - Muy bueno } \\
& \text { - Bueno } \\
& \text { - Regular } \\
& \text { - Malo }
\end{aligned}
$$

Fuente: Elaboración propia 
Análisis: Con respecto al confort que prestan las unidades de transporte, el 54\% manifiestan que es malo, el $25 \%$ que es regular, el $12 \%$ que es bueno y el $9 \%$ de usuarios manifiesta que es muy bueno o le brinda todas las condiciones que requieren.

Pregunta 12. El manejo de las unidades de transporte (Bus), por parte de los choferes es:

Gráfico 12. Manejo adecuado de las unidades de transporte.

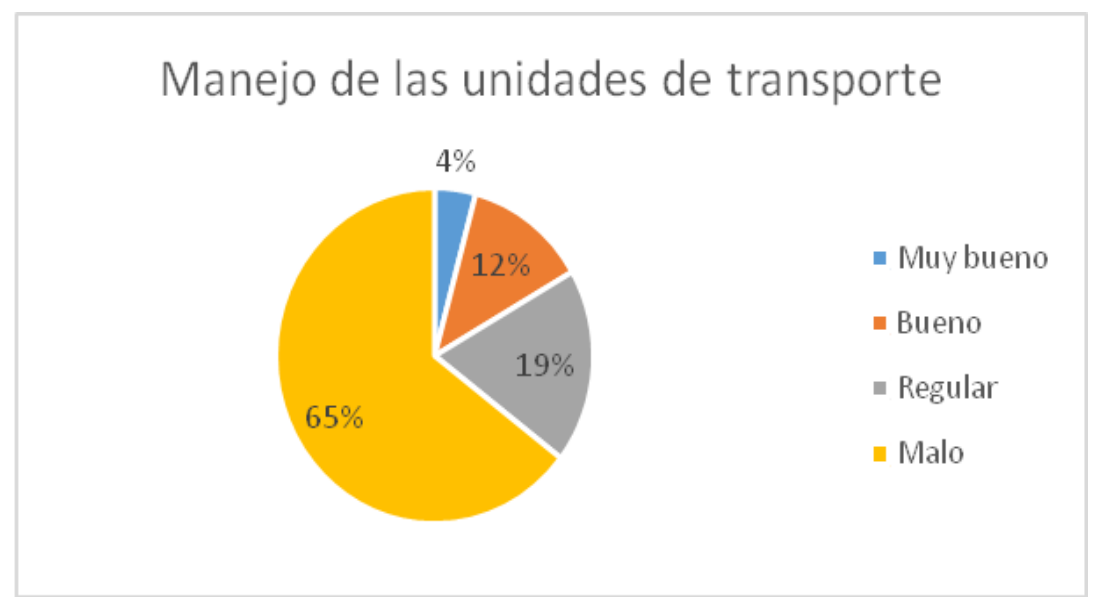

Fuente: Elaboración propia.

Análisis: Del total de encuestados, el 65\% manifiestan que el manejo de las unidades de transporte por parte de los choferes es malo, el 19\% cree que es regular, el $12 \%$ manifiesta que es bueno, y el $4 \%$ manifiesta que es muy bueno.

\section{Seguridad.}

Pregunta 13. La seguridad en contra agresiones en los buses es:

Gráfico 13. La seguridad contra agresiones.

La seguridad contra agresiones en los buses

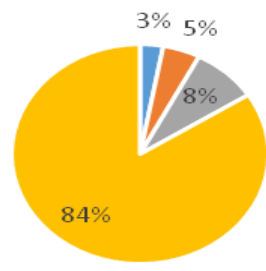

- Muy buena

- Buena

- Regular

- Mala

Fuente: Elaboración propia. 
Análisis: el $84 \%$ de usuarios del servicio de transporte cree que la seguridad en contra de agresiones en el bus es mala o no existe, el $8 \%$ manifiesta que es regular, el $5 \%$ que es buena, y el $3 \%$ que es muy buena la seguridad al interior del bus.

\section{Impacto ambiental.}

Pregunta 14. Las unidades que operan actualmente en el servicio de transporte generan contaminación ambiental, como emisión de gases, ruido excesivo, olores, etc.

Gráfico 14. Contaminación ambiental.

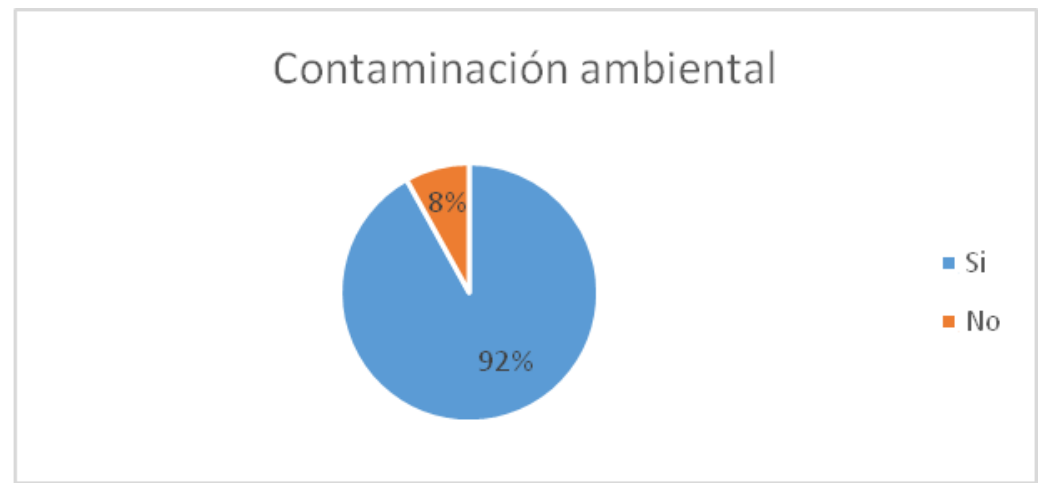

Fuente: Elaboración propia

Análisis: Del total de encuestados, el 92\% manifiestan que las unidades que operan actualmente en el servicio de transporte generan contaminación ambiental, mientras que el $8 \%$ restante cree que los buses no generan contaminación.

Nivel de satisfacción de los usuarios del servicio de transporte urbano de buses de la ciudad de Ambato.

El nivel de satisfacción de los usuarios del servicio de transporte urbano en buses de la ciudad de Ambato se midió aplicando la fórmula de Thomson (2005), qué expresa, que el nivel de satisfacción es igual al rendimiento percibido menos las expectativas.

Se considera una expectativa como elevada cuando el porcentaje de referencia que indicaron los usuarios el servicio supere el $50 \%$, si el porcentaje se ubica entre el $20 \%$ y $50 \%$ la expectativa será moderada, y en el caso de ser inferior al $20 \%$, será considerada como baja.

Las principales variables consideradas para medir el nivel de satisfacción de los usuarios fueron Accesibilidad, Información relativa al viaje, tiempo en ruta, Atención al cliente, Confort, seguridad. 
Otras variables importantes que no fueron consideradas para medir el nivel de satisfacción de los usuarios debido a la dificultad que para esto representa esta medición son impacto ambiental y servicio ofertado. Sin embargo, en la discusión se incorporan estas variables con la finalidad de presentar los diferentes criterios estudiados.

En torno a este enunciado se detallan en la tabla 2 los indicadores del nivel de satisfacción del servicio de bus, cabe indicar que la media de nivel de satisfacción es igual a 2,93 sobre 10 .

Según estos indicadores, la prioridad del servicio o las variables de calidad que el usuario considera de mayor importancia son la Atención al cliente, el Confort, la Seguridad que le da viajar en un bus; La información relativa al viaje, El tiempo en ruta y la accesibilidad.

Tabla 2: Indicadores del nivel de satisfacción del servicio de Transporte en bus.

\begin{tabular}{|c|c|}
\hline VARIABLES & Nivel de Satisfacción (/10) \\
\hline Accesibilidad & $\mathbf{2 , 8 9}$ \\
\hline $\begin{array}{l}\text { La accesibilidad que proporcionan los buses, para niños, } \\
\text { mujeres embarazadas, adultos mayores y discapacitados es }\end{array}$ & $\mathbf{2 , 8 9}$ \\
\hline $\begin{array}{c}\text { Información } \\
\text { La información relativa al viaje, que le proporcionan en las } \\
\text { paradas y en los buses es }\end{array}$ & $\mathbf{3 , 6 7}$ \\
\hline $\begin{array}{l}\text { Tiempo } \\
\text { El tiempo que tarda en su recorrido (Ruta) desde su origen al cliente } \\
\text { a su destino es }\end{array}$ & $\mathbf{1 , 5}$ \\
\hline El trato recibido por parte de choferes y cobradores es: & $\mathbf{3 , 7}$ \\
\hline $\begin{array}{l}\text { El confort que prestan las unidades de servicio de } \\
\text { transporte a los pasajeros es. }\end{array}$ & $\mathbf{3 , 2 9}$ \\
\hline $\begin{array}{l}\text { El manejo de las unidades de transporte (Bus), por parte de } \\
\text { los choferes es }\end{array}$ & \\
\hline \multicolumn{2}{|c|}{ Seguridad } \\
\hline La seguridad en contra agresiones en los buses es
\end{tabular}

Fuente: Elaboración propia

Si analizamos la tabla 2, podemos mencionar que el nivel de satisfacción por parte de los usuarios del sistema de transporte público de la ciudad de Ambato es relativamente bajo, si se considera la escala de Tomson, que dice: 
Una puntuación entre 8 y 10 el cliente está complacido, entre 5 y 7 el cliente está satisfecho y un valor igual o menor a 4 es un cliente insatisfecho.

Analizando al detalle, se puede mencionar que los usuarios del servicio de transporte urbano en bus de la ciudad de Ambato, en la variable accesibilidad tienen un nivel de satisfacción del 2,89 sobre 10 lo que implica que en esta variable los usuarios están insatisfechos.

En lo que corresponde a la variable información el nivel de satisfacción es de 2,89 lo que implica que los usuarios del servicio de transporte urbano en bus están insatisfechos con respecto a este tema.

En la variable tiempo se puede apreciar que el nivel de satisfacción es de 2,67 por lo tanto el nivel de satisfacción del usuario en promedio es insatisfecho.

La variable Atención al Cliente, es la más baja de toda la tabla llegando a un nivel de satisfacción de 1,5 sobre 10 lo que significa que la cliente esta, insatisfecho.

Si analizamos el confort que prestan las unidades de transporte, se alcanza un 3, 7 sobre 10 lo que implica que el usuario está inconforme o insatisfecho, y por último en la variable seguridad se alcanza un nivel de satisfacción de 2,6 qué representa una insatisfacción por parte del usuario.

\section{Conclusiones.}

- Para medir la calidad del servicio de transporte se aplicó los criterios de calidad según la norma europea UNE-EN 13816, estas variables, nos permitieron determinar el nivel satisfacción de los usuarios del servicio de transporte urbano de Ambato, llegando a determinar que en las ocho variables estudiadas el nivel de satisfacción esta, por debajo de 4 sobre 10; lo que implica que los usuarios están insatisfechos con el servicio de transporte en bus.

- Los resultados de la investigación evidencian ampliamente la situación actual del sector de la transportación, y la consecuente necesidad de trabajar en procesos de corto, mediano y largo plazo en la temática, con objetivos claros y concisos que aporten en cambios de comportamiento, exigiendo la implementación de estrategias de mejora y un continuo monitoreo y evaluación de los impactos de dichos procesos.

- El Gobierno Autónomo Descentralizado de Ambato, debe ser quien se apodere de la situación, y se emprenda en procesos de solución inmediatos, reprogramando rutas, y brindando mayor cobertura, así como también con el estudio de la flota vehicular, 
adecuación de vías y señales de tránsito y por otro lado exigiendo a las operadoras de transporte urbano en bus la corrección de sus procesos para enmendar las falencias detectadas en el servicio que prestan.

\section{Referencias bibliográficas.}

Arroyo, A. C. (2008). La política común de transportes: origen y evolución en Europa del transporte público por carretera. Anuario Jurídico y Económico Escurialense, XLI, (págs. 49-68).

BCE, B. C. (2013). Estadisticas Macroeconomicas. Quito: BCE.

Censos, I. N. (2010). Proyecciones de la población ecuatoriana por años, según cantones. Quito: INEC.

Comité Europeo de Normalización. (2006). Criterios de calidad según la norma UNE-EN 13816. Obtenido https://upcommons.upc.edu/bitstream/handle/2099.1/5960/13.pdf?sequence=15\&is Allowed=y

Dombriz, M. A. (2009). Urbanismo y movilidad: dos caras de la misma moneda. Revista Ingeniería y Territorio, núm. 86, 4-9.

Domínguez, H. (2006). El servicio invisible : fundamento de un buen servicio al cliente. Bogotá: Ecoe Ediciones.

Figueroa, O. (2005). Transporte urbano y globalización. Políticas y efectos en América Latina. Eure, 41-53.

Fundación CETMO. (2006). Ministerio de Fomento España. Norma Europea. Obtenido de Manual de Apoyo para la Implantación de la Gestión de la calidad según Norma UNE-EN 13816: https://www.fomento.gob.es/recursos_mfom/pdf/6336A7393BBF-426C-AD69-

1F3C59833A19/25583/Cap1ElmodelodecalidadsegunUNEEN13817.pdf

Gutiérrez. (2008). Administrar para la calidad: conceptos administrativos del control total de la calidad. México: Limusa S.A de C.V.

Gutiérrez. (2010). Calidad total y productividad. México: Editorial Mc Graw-Hill.

Gutiérrez, A. (2009a). Movilidad o inmovilidad: ¿Qué es la movilidad?. Aprendiendo a delimitar los deseos. In XV Congreso Latinoamericano de Transporte Público y Urbano. [CD-Rom]. Buenos Aires.

Gutiérrez, A. (2010). Movilidad, transporte y acceso: una renovación aplicada al ordenamiento territorial. Revista electrónica de geografía y ciencias sociales. Vol. XIV, núm. 331 <http://www.ub.es/geocrit/sn/sn-331/sn-331-86.htm>, 4.

Hernández, R. (2011). Fundamentos de gestion empresarial. Mexico: Mc Graw Hill.

Hubenthal, A. (2010). Evaluación del sector transporte en Ecuador con miras a plantear medidas de mitigación al Cambio Climático. Quito: Programa de la ONU.

INEC. (2010). V Censo Poblacional y Vivienda. Quito: INEC. 
MDMQ. (2009). Municipio del Distrito Metropolitano de Quito. Plan MAestro de Movilidad para el Distrito Metropolitanode Quito. Obtenido de file://C:/Users/C\&DSYSTEMS/Downloads/LFLACSO-01-DMQ.pdf

Morán, G. (2016). ANÁLISIS A LA CALIDAD DEL SERVICIO DE TRANSPORTE URBANO DE BUSES EN LA CIUDAD DE ESMERALDAS DESDE LA ÓPTICA DEL USUARIO. Esmeraldas. Obtenido de https://repositorio.pucese.edu.ec/bitstream/123456789/702/1/MORAN\%20VILLAF UERTE\% 20\%20GABRIEL.pdf

MTOP. (2010). Manual para estudios de origen y destino de transporte de pasajeros y mixto en áreas municipales distritales y metropolitanas. Quito: Registro Oficial.

Publicas, M. d. (2009). Estadisticas del Transporte en el Ecuador. Quito.

Silva, N., \& Torres, C. (2017). Calidad del servicio del transporte urbano en la ciudad de Cuenca. Cuenca. Obtenido de https://dspace.ups.edu.ec/bitstream/123456789/14263/1/UPS-CT007011.pdf

Thomson, I. (2005). La satisfacción del cliente. Obtenido de http://moodle2.unid.edu.mx/dts_cursos_mdl/pos/MD/MM/AM/03/Satisfaccion_del _Cliente.pdf

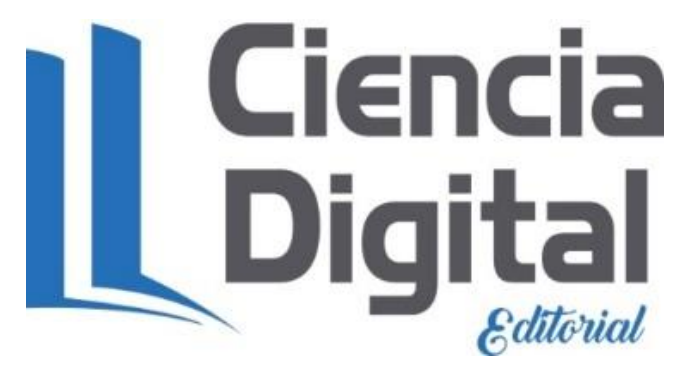


Para citar el artículo indexado.

Llamuca J. \& Aguilar G. (2019). Evaluación de la calidad del servicio de transporte urbano en bus de la ciudad de Ambato., Revista electrónica Ciencia Digital 3(2), 26-46. Recuperado desde: http://www.cienciadigital.org/revistascienciadigital/index.php/VisionarioDigital/article/view/392/87 $\underline{6}$

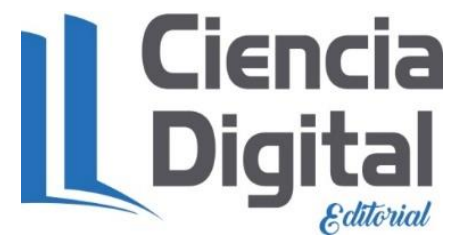

El artículo que se publica es de exclusiva responsabilidad de los autores y no necesariamente reflejan el pensamiento de la Revista Ciencia Digital.

El artículo queda en propiedad de la revista y, por tanto, su publicación parcial y/o total en otro medio tiene que ser autorizado por el director de la Revista Ciencia Digital.
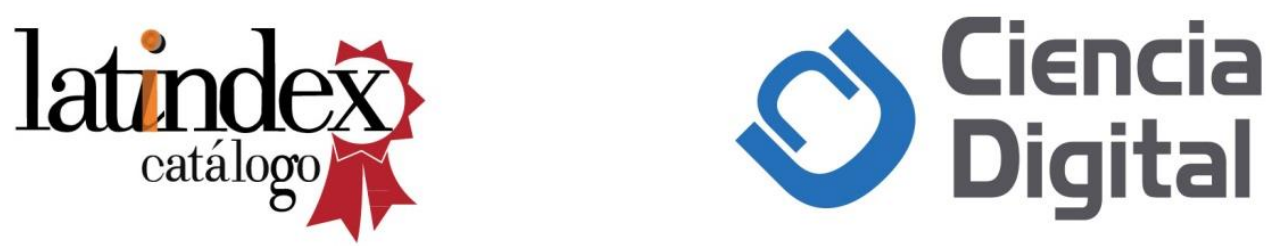